\title{
Communication in Multidisciplinary Teams
}

\section{Snezana Medakovic}

Specialist Scrub Nurse, Institute for Cardiovascular Diseases Dedinje, Serbia

Corresponding author: Elharrouni Alaoui Aicha, Department of dermatology, CHU Hassan II, FES, Morocco. E-mail: ealaouiaicha@gmail.com

Received: February 24, 2020; Accepted: March 02, 2020; Published: March 06, 2020.

Keywords: Marjolin's ulcer, Chronic Radiodermatitis, Chronic skin disease.

"No one can say so much wisdom, how much can hesitate to crazy." Jovan Ducic The legend says: God demolished Babylon Tower, by making it to builders and he mixed up the languages so that building would not reach heavenly heights. People constantly build and demolish a civilization tower, creating a new technical, technological, professional and scientific languages. The result is that nobody understands anybody anymore. On the one hand, natural native languages die one by one, every fifteen days, and on the other hand every fifteen days there is a new language of a new technology and branches of science one by one.

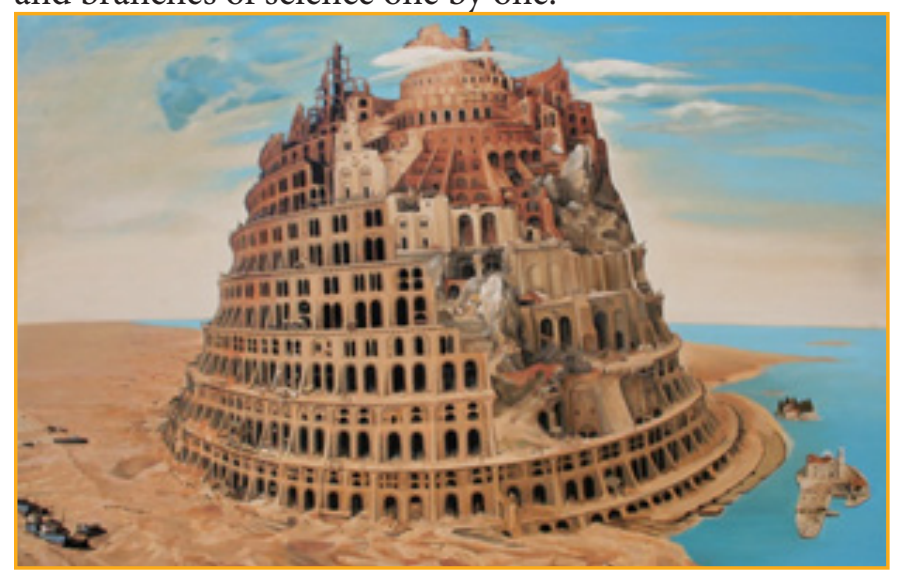

Figure1: Babylon Tower .

Communication among people is like atomic energy, it can destroy the world, but it can lead to incredible progress. When we say: good communication, then we think make that communication between people must not allow it to have stronger and weaker sides, respectively neither party should be overwhelmed by the authority of another person. In the conversation of two human beings each side needs to get on he quality of their personality. People need to learn wisdom, happiness, love, faith, charisma, knowledge, art, as is learned and exercises the power of conversation.

One of the main and leading problems in the work of many professionals, even among health professionals is a matter of communication. We live in a time when many technical and technological possibilities are available to us to send information quickly to all users, the general impression is that our quality of communication at a very low level and that it is very often present at all to the levels from which it should be forwarded to other team members.

The success and quality of the team depend, in the first place, from the existence of good communication. Successful and good communication is the basis of all further activities and which ensures successful 


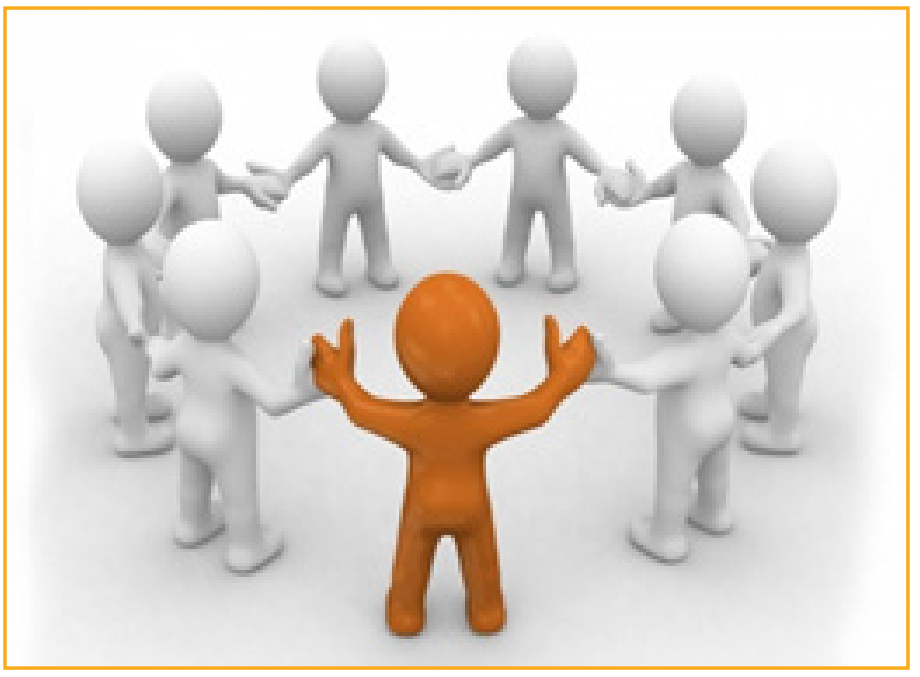

Figure 2: Good communication.

When we are talking way is important good communication in a medical team, we need to look a few aspects.

- Communication is the basis of every relationship among people

- The way of communication we are talking about ourselves to other people

- By good communication we achieve a better relationship and confidence of patients

- We achieve a better relationship with other associates, as well as a pleasant atmosphere at the workplace

- We will achieve better results of work and health care for the patient

- - Greater personal satisfaction at the work we do

- - Very often the characteristic of good communication is kindness, and she must be an integral part of our professional work. Way that is important? When we are thinking about kindness, we must to know what we achieve:

- We achieve our personal development and maturation

- - We improve personal and professional relationships

- Daily work in a healthy and efficient professional team, and it encourages employee satisfaction and provides better time management

- Resolving disagreements and conflict increases the complexity of the team

- For a medical team it's very important to achieve certain preconditions for successful work in the team. That it's reasons why I want to list them:

- $\quad$ Listening

- Work reports

- $\quad$ Regular meetings
- $\quad$ Accepting differences in opinion

- $\quad$ Adherence to the agreement

- $\quad$ Respect

- Openness

- Support

- Trust

All this is very important to create a harmonized teamwork in health care, and that way we encourage patients to better cooperate. The emergence of a computer network has created a completely new form of communication in relation to the existing, intrapersonal, as well interpersonal communication in larger social groups and mass communication. Although many condemn social networks, because people in communication speak about the most intimate parts of their lives, they don't necessarily have to be bad. There are also such social networks in which they are in various interests groups, you can hang out and share experiences as well as get the necessary information from your profession.

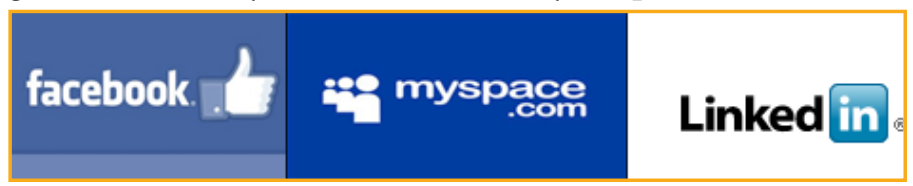

Figure 3: Social networks.

In 2015 was conducted a discussion on the social network: "Nurses eat their young...Are you agree with this ?"

Three responses were offered:

- I agree-108-76\%

- I do not agree $11-7 \%$

- maybe $23-17 \%$

The discussion lasted for two months, there were a total of 40 participants, the average age of the subjects was $25-55$, almost nurses around the world. These devastating results indicate that the relations of a nurse-nurse, especially those bad are characteristic for all countries and for all environments. Through the answers in this discussion, can lead to a conclusion that there are similar and universal problems:

1. Unequal struggle and unfair competition

2. Senior nurses have a passive attitude towards doctors, and aggressive towards with a new nurse

3. Hiatus among the generations, a glance at the past and the difficult adoption of new doctrines by more experienced nurses

4. The assertive attitude of new nurses, more confidence and unwillingness to listen to stories from the past

5. Experienced nurses who have a passive attitude 
towards doctors come to the leadership positions, and then they show aggression towards colleagues

6. Jealousy, as a very present emotion (for any reason)

7. Experienced nurses emphasize their experience, and a new nurse by knowing new technologies and faster readiness to acquire new knowledge

The general conclusion of this discussion is that the family environment and education, as well as the culture of the environment, contribute to a poor relationship among the nurses. Everywhere in the world, nurses are a large population group, when they were complicated and when they agreed on attitudes could do a lot for the profession and for their personal affirmation.

What is important to know how to improve interpersonal and professional relationships in the nurse's team? In the first-place communication a nurse-nurse, especially if the relationship is senior-younger nurses, it is very often characterized by this that there is a generation conflict in communication, due to unresolved competencies and job distribution. Two nurses of the same educational level, but with differences in years, they should follow the old rules: that the younger respect the elderly, but also that they respect younger.

Communication manager-nurse, for this type of communication, a significant style of leadership as well as a good division of indebtedness. The department manager is obliged to inform the nurses about their duties. In these relationships it is very important to have an assertive style of behavior, what does it mean to occupy and defend your attitudes, but that they don't endanger other rights and attitudes.

Communication nurse-doctor, this aspect of communication should take place openly, which means that a nurse should always show his competence through precise assessments and quality performance of work assignments. Here is the preferred form of communication-assertiveness. Nurses in communicating with doctors must have developed selfesteem, because if a nurse doesn't appreciate herself, others will not appreciate it.

Nursing is by her own nature-based cooperation. Nurses and other medical staff must have effective communication in order to provide adequate patient care. Good communication prevents the occurrence of errors or at least reduces their degree. Nurses need to develop a high degree of self-esteem, and this will be reflected in the respect of others. They must be constantly educated and thus gain their own safety and respect for their patients, associates and employers. The nurse is part of a medical team in which it is necessary to participate equally, but this is only possible if he knows his job well. Any inconsistency destroys self-esteem. The strongest weapon of nurses is knowledge.
Copyright: (C2020 Snezana Medakovic. This is an open-access article distributed under the terms of the Creative Commons Attribution License, which permits unrestricted use, distribution, and reproduction in any medium, provided the original author and source are credited. 